\title{
Influence of surface roughness and deposition order on exchange bias in bilayer structures NiFe/IrMn
}

\author{
E. I. Shanova ${ }^{1,2}$, I. O. Dzhun ${ }^{2}$, N. G. Chechenin ${ }^{1,2, a}$ \\ ${ }^{1}$ Faculty of Physics, Lomonosov Moscow State University, Leninskie Gory 1/2, Moscow 119991, Russian \\ Federation \\ ${ }^{2}$ Skobeltsyn Institute of Nuclear Physics, Lomonosov Moscow State University, 1/2, Leninskie Gory, Moscow \\ 119991, Russian Federation
}

\begin{abstract}
Using ferromagnetic resonance (FMR) method, magnetic properties of bilayer structures consisting of ferromagnetic $\left(\mathrm{F}, \mathrm{Ni}_{80} \mathrm{Fe}_{20}\right)$ and antiferromagnetic $\left(\mathrm{AF}, \mathrm{Ir}_{25} \mathrm{Mn}_{75}\right)$ layers with alternative order of $\mathrm{F}$ and $\mathrm{AF}$ deposition were studied. It was found that the surface roughness is larger for the samples with $\mathrm{AF}$ on the top of $\mathrm{F}$ layers. The influence of AF layer thickness on the surface roughness and exchange bias for different types of structures was discussed.
\end{abstract}

\section{Introduction}

Bilayer ferromagnetic/antiferromagnetic (F/AF) structures are basic elements in many magnetic devices, therefore their properties received a considerable interest both in theoretical context and application. Exchange interactions at F-AF interface in the structures lead to an unidirectional magnetic anisotropy, manifesting itself as a shift in the hysteresis loop by an amount $H_{E B}$ called exchange bias [1].

A major portion of publications shows that exchange bias shift decreases when roughness increases [1-4], though no significant correlations between $H_{E B}$ and roughness was found in some of the works $[5,6]$. An opposite effect, that is $H_{E B}$ increasing when roughness increased, was also demonstrated in other investigations, $[1,3,7]$, for instance, in monocrystalline silicone $\mathrm{AF}$ (with compensated and uncompensated surface), covered with an F-layer. This controversy indicates the important role of the interface surface morphology in appearance of magnetic properties.

In case of uncompensated AF layer, the decreasing of $H_{E B}$ with increasing roughness can be attributed to the emergence of areas with alternately oriented spins on F/AF interface, which inevitably leads to a decrease in the number of spins, directed in the biasing direction, causing a weakening of the total unidirectional exchange bias field. Compensated interfaces with high roughness, either might still be kept statistically compensated in average, leaving the exchange bias unchanged or, alternatively, became uncompensated if the statistical variation of spins orientation will be shifted under influence of magnetization of an underlying $\mathrm{F}$ layer, thus making $H_{E B}$ enhanced.

The investigation of factors influencing exchange bias, aside from fundamental issues of relationship between structural and magnetic properties in multilayered systems, is also of great practical value, because formation of such systems is carried out under various conditions, which leads to variation in structural and compositional characteristics. The range of variation of such characteristics determines quality and cost of devices that are using spintronics and magnetic sensors.

In this paper, we investigate how exchange bias is affected by AF layer thickness, by the order of deposition and surface roughness. Exchange bias is measured using ferromagnetic resonance (FMR) angular distribution. Finally, we discuss possible explanations for the features we observed.

\section{Samples preparation and measuring}

The samples were prepared, using a DC magnetron sputtering, as 4-layer structures $\mathrm{Ta} / \mathrm{NiFe} / \mathrm{IrMn} / \mathrm{Ta}$ (TS) and $\mathrm{Ta} / \mathrm{IrMn} / \mathrm{NiFe} / \mathrm{Ta}(\mathrm{BS})$ on silicon substrate. The tantalum layers with the thickness of $30 \mathrm{~nm}$ served as buffer and protection layers. Two sets of the samples were made: one set with IrMn AF layer at the top of $\mathrm{NiFe} F$ layer (TS), the other one with $\mathrm{AF}$ layer underneath the $\mathrm{F}$ layer (BS). Both sets had the same series 10,20,30 and $40 \mathrm{~nm}$ of the AF thickness. The thickness of the F layers was $10 \mathrm{~nm}$, the same for all the samples.

The base pressure in the sputtering chamber was $10^{-7}$ torr, the argon pressure during the deposition was kept around

\footnotetext{
${ }^{\mathrm{a}}$ Corresponding author: chechenin $@$ sinp.msu.ru
} 
2.5.mtorr. $\mathrm{Ni}$ and $\mathrm{Fe}$ targets were used to grow the $\mathrm{Ni}_{80} \mathrm{Fe}_{20}$ layer and $\mathrm{IrMn}$ target to produce the $\mathrm{Ir}_{25} \mathrm{Mn}_{75}$ layers. The proper layer composition, as well as the layers thickness, was obtained specifying the DC power, hence, the deposition rate, and sputtering time for each target and each layer, basing on calibrations made using the dummy samples and the Rutherford Backscattering Technique. The deposition rate was for $\mathrm{NiFe}$ layers and $3.7 \mathrm{~nm} / \mathrm{min}$ for IrMn layers. It is important to note for further consideration that all the layers for all the samples were deposited at the same rate of $3.7 \mathrm{~nm} / \mathrm{min}$.

Magnetic order in the AF layer was induced during the deposition in a magnetic field of $420 \mathrm{Oe}$ in plane of the substrate supplied by permanent magnet. Above this, the growth of the AF layer in the TS samples was influenced by magnetization of the $F$ layer saturated in the external deposition field.

The magnetic characteristics were investigated with FMR with fixed field frequency of $9,65 \mathrm{GHz}$. Saturation magnetization $M_{S}$ and uniaxial magnetic anisotropy field $H_{K}$ can be obtained from resonance field value $H_{r}$, directed along easy axis (EA) and hard axis (HA) using the Kittel equations [8]:

$$
\begin{aligned}
& \omega^{2}=\gamma^{2}\left(H_{r}^{E A}+H_{K}\right)\left(H_{r}^{E A}+H_{K}+4 \pi M_{s}\right) \\
& \omega^{2}=\gamma^{2}\left(H_{r}^{H A}-H_{K}\right)\left(H_{r}^{H A}-H_{K}+4 \pi M_{s}\right)
\end{aligned}
$$

Here $\omega=2 \pi f$ is the frequency of the microwave field, $\gamma=g e /(2 m c) \approx g \cdot 8.79 \cdot 10^{6}(G \cdot s)^{-1}$ - gyromagnetic ratio, $H_{r}{ }^{E A}, H_{r}{ }^{H A}$ resonance fields applied along easy and hard axes.

These equations can be extended to the case of any orientation of the external FMR field (relative to the uniaxial magnetic anisotropy of a single ferromagnetic layer), as well as to the case of unidirectional anisotropy in the F-layer, caused by exchange bias in the binary system on F-AF interface.

If exchange bias field is parallel to EA of the F-layer form the angle $\theta$ with the direction of the external field, then for $4 \pi M_{S} \gg H_{r}$ field $H_{r}$ is determined by the intrinsic resonance field $H_{r O}=(\omega / \gamma)^{2} /\left(4 \pi M_{S}\right)$ of the Flayer and values of $H_{K}$ and $H_{E B}$ and angle $\theta$, according to the expression $[9,10]$ :

$$
H_{r}=\frac{\omega^{2} / \gamma^{2}}{4 \pi M_{s}}-H_{E B} \cos \theta-H_{K} \cos 2 \theta
$$

Since EA direction in our experiment was defined by the field direction during deposition, $\theta$ is the angle between permanent FMR field and deposition field.

Sample surface morphology was investigated by atomic force microscope Integra Spectra (NT MDT) (AFM), using semicontact mode at $\Delta \mathrm{Z}=20 \pm 5 \mathrm{~nm}$ in the second passage. The mean square roughness $\sigma_{\mathrm{rms}}=<\Delta \mathrm{h}^{2}>^{1 / 2}$ was obtained in this way, where $\Delta \mathrm{h}$ is the deviation of the surface at given point of the scan from the average value.

\section{Results}

The dependences of exchange bias $H_{E B}$ on AF layer thickness in TS and BS samples are plotted in Figure 1. Maximum bias value is reached at $t_{A F}=30 \mathrm{~nm}$, while lower thickness gives lower $H_{E B}$. At $t_{A F}=10 \mathrm{~nm}$ in BS, $H_{E B}$ still decreases, while in TS exchange bias rises again. Note that conditions of exchange bias forming in TS and BS are different. In TS samples AF layer was formed on magnetically saturated $F$ layer under appropriate strong magnetic field. In BS samples AF layer grows under comparatively weak external permanent magnetic field. The subsequent $F$ layer is not able to change the micromagnetic order of the AF layer. Hence, the presence of exchange bias in BS samples means that a comparatively weak external field is sufficient to form a unidirectional magnetocrystalline anisotropy in the AF layer.

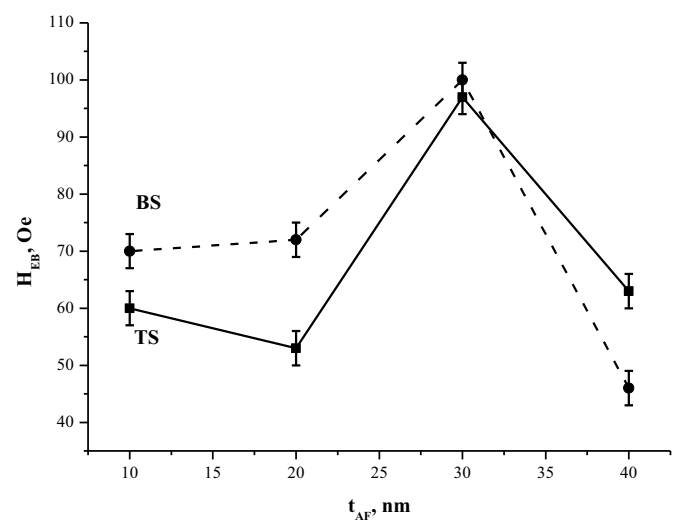

Figure 1. Dependence of the exchange bias in TS and BS on AF layer thickness

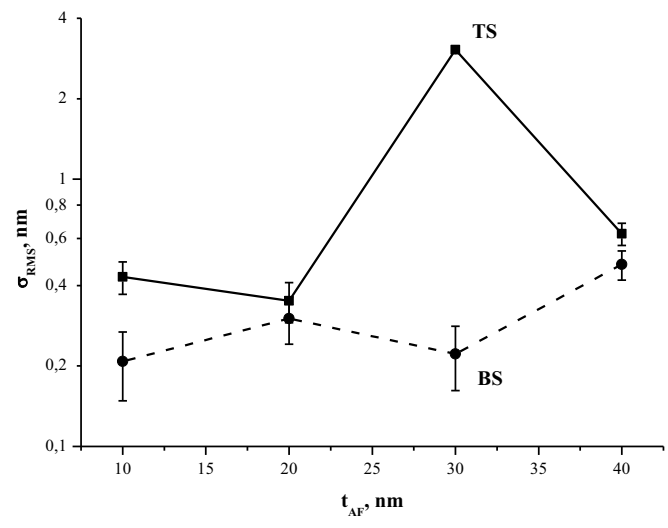

Figure 2. Root-mean-square roughness for TS and BS as a function of $\mathrm{AF}$ thickness

Figure 2 presents a root-mean-square roughness $\sigma_{\text {rms }}$ and AF layer thickness $t_{A F}$ relationship for TS and BS samples. This dependency is nonmonotonous for both structure types. The variation of $\sigma_{\mathrm{rms}}$ with the sample thickness is opposite for TS and BS. TS samples, however, have significantly higher roughness values.

A direct relation between surface roughness and exchange bias is illustrated in Figure 3. Despite the 
difference in TS and BS roughness range, the exchange bias range is alike. However, any correlation between $H_{E B}$ and $\sigma_{\text {rms }}$ is absent as it follows from the figure at first sight. This, however, is an oversimplified view. We shall revisit this issue in the next section.

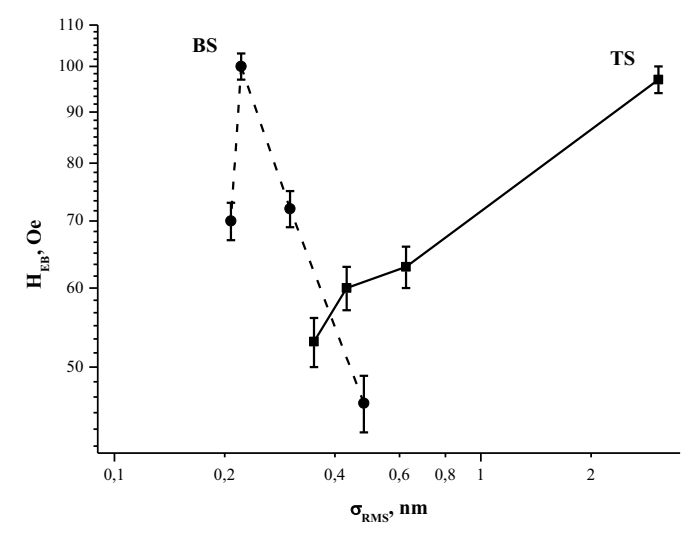

Figure 3. Exchange bias dependency on roughness for TS and BS samples

\section{Discussion}

\subsection{Exchange bias}

The dependence of exchange bias on AF layer thickness was investigated in a number of papers for bilayer $\mathrm{F} / \mathrm{AF}$ and trilayer $\mathrm{F} 1 / \mathrm{AF} / \mathrm{F} 2$ systems for different combinations of $\mathrm{F}$ and $\mathrm{AF}$ layers, their structure and composition. A steep $H_{E B}$ decrease for lower thickness $t_{A F}$ is a general tendency in those findings. Moreover, for $t_{A F}$ lower than some critical $t_{\mathrm{C}}$ exchange bias did not emerge. The critical value $t_{\mathrm{C}}$ depends on $\mathrm{AF}$ and $\mathrm{F}$ layer properties. This tendency can be interpreted as a manifestation of size effect, that leads to a blocking temperature decrease relative to Neel temperature for lower thickness [11] and thus decreases crystalline anisotropy energy $K_{\mathrm{AF}}$ and exchange bias field. At $t_{A F}<t_{\mathrm{C}}$, the energy density per interface area becomes less than exchange energy of the F/AF pair, $K_{\mathrm{AF}} \cdot t_{A F}<K_{\mathrm{AF}} \cdot t_{C}=J_{I N T}$. In this case magnetic moment of AF on the interface will follow the magnetic moment of $\mathrm{F}$, i.e. there will be no bias. At $t_{A F}>$ $t_{\mathrm{C}}, H_{E B}$ grows with increasing thickness and reaches maximum at $t_{A F}=t_{\max }$, and at still higher thickness $H_{E B}$ either saturates or decreases [12-17].

In Malozemoff model [18] AF layer thickness range is divided into three sub-ranges. Exchange bias emerges when exchange energy on F/AF interface is high enough to form a domain structure with domain walls oriented normally to the interface. The domains are of finite size, so that they produce a randomly oriented field, but its average per domain area, however, does not vanish to zero even for compensated and unsmooth interfaces. At some thickness domain wall energy and exchange energy become equal and bias emerges at $t_{\mathrm{AF}}<\mathrm{t}_{\mathrm{AF}, 1}$. At $t_{\mathrm{AF}, 2}<t_{\mathrm{AF}}<t_{\mathrm{AF}, 1}$ domain size and, accordingly, exchange bias field remain almost the same. At $t_{\mathrm{AF}}<t_{\mathrm{AF}, 2}$ domain size decreases, and $H_{E B}$ increases with decreasing $\mathrm{AF}$ thickness until a certain value of $t_{\mathrm{AF}, 3}$ is reached. At $t_{\mathrm{AF}}<t_{\mathrm{AF}, 3}$ domain structure becomes unstable, domain walls follows reversing magnetization in the $\mathrm{F}$ layer, $H_{E B}$ faces a severe decrease to zero.

A similar approach has been recently developed in a series of papers by the scientific group in York (see for example a review [19]). This approach is based on the idea that exchange bias emerges as a consequence of grains in AF layer, that have their size within a certain range. Too small grains are thermally unstable and thus are not able to provide the necessary anisotropy energy to remain stable when external field is reversed. Too large grains have their anisotropy energy so high that too strong field is required to create a unidirectional anisotropy in them. In their work [20], the authors showed that concentration of grains with volume within the optimal range (and, consequently, exchange bias) is a function of AF layer thickness.

The results we obtained for $H_{E B}$ dependency BS are in agreement with Malozemoff model [18] and York model [19]: $H_{E B}$ grows at lower thickness range, then reaches its maximum value, ant then decreases. But in TS systems at $10 \mathrm{~nm} H_{E B}$ behavior contradicts such pattern $-H_{E B}$ grows instead of decreasing. Clearly, models $[18,19]$ do not take into account other important mechanisms of exchange bias initiation in F/AF system.

Among such mechanisms, above all, one should consider grain size variation, texture and roughness change with AF layer thickness and F-AF layer order.

\subsection{Roughness in TS and BS}

The evidence presented on Figure 2 shows that roughness values for TS systems, though being nonmonotonous, significantly exceeds roughness in BS systems. Attention is also drawn to the fact that TS and BS dependencies are acting in an opposite way. The disparity begins from thickness $t_{A F}=10 \mathrm{~nm}$. In this case TS and BS have the same thickness, and the only difference is in F-AF layer order. The fact that the samples showed different resulting roughness suggests that TS and BS systems have different layer growth mechanism due to alternating deposition order.

One should also notice that roughness change with AF thickness in TS systems correlates with exchange bias change (compare Figure 1 and 2). Increase in $\sigma_{\mathrm{rms}}$ corresponds to $H_{E B}$ increase and vice versa. This implies that 1) roughness, does make an essential contribution to the exchange bias; 2) roughness, measured on the sample surface does correspond to the one of the F/AF interface, thus providing uncompensated magnetic moment in the boundary layer, and 3) comparing TS and BS roughness, one can conclude, that surface roughness is a competition of two competing processes with alternating success when the thickness growths, that are enhancing and smoothing of the surface modulation. Basing on the Figure 2 one can conclude that the period of the roughness variation with the thickness is of $20 \mathrm{~nm}$ which, probably, corresponds to the AF grain size. And for this to add, at smallest AF thickness $10 \mathrm{~nm}$ 
smoothing dominates for BS and roughening - for TS samples.

In contrast to TS, BS samples do not show an evident correlation between roughness and exchange bias, compare Figure 1 and 2 . Taking into account that $\sigma_{\mathrm{rms}}$ is much smaller in BS compared to TS, we assume that the roughness is simply too small to play a significant role, while AF grain size variation can affect to a larger extent via mechanisms discussed in the previous section.

Putting it all together, exchange bias value is determined by several factors. Variations of $H_{E B}$ in BS are to a great extent defined by grain size changing with thickness, and in TS systems they are defined by differences in F-AF interface roughness.

\section{Conclusions}

1) We have studied exchange bias dependencies of $H_{E B}$ on root-mean-square surface roughness $\sigma_{\mathrm{rms}}$ for samples in alternative $\mathrm{F}$ and $\mathrm{AF}$ layers deposition order in $\mathrm{Ni}_{80} \mathrm{Fe}_{20} / \mathrm{Ir}_{25} \mathrm{Mn}_{75}$ bilayer structures for different thickness in the 10-40 $\mathrm{nm}$ range.

2) We observed that exchange bias takes close values for TS (AF over F) and BS (AF under F) systems and changes with thickness of the AF layer synchronously.

3) The value of $\sigma_{\text {rms }}$ in TS significantly exceeds the one in BS samples, however, the variations of $\sigma_{\text {rms }}$ with AF thickness in TS and BS show opposite tendencies.

4) The observed distinctions in the samples with alternative order of deposition of $\mathrm{F}$ and AF layers referred to microstructure being formed in a different manner in TS and BS systems, which leads to variations in surface topography, grain size, texture and resulting in different extent of AF layer spins compensation and $\mathrm{AF}$ anisotropy.

\section{Acknowledgement}

This work is supported by Russian Foundation for Basic Research (contract 12-02-31541) and by Ministry of Science and Education of RF via g/c 14.513.11.0116. The FMR study was performed at User Facilities Center of M.V. Lomonosov Moscow State University.

\section{References}

1. J. Nogués and I. K. Schuller, J. Magn. Magn. Mater, 192, 203 (1999)

2. D. Lederman, J. Nogués, and I. K. Schuller, Phys. Rev. B, 56, 2332 (1997)

3. J. X. Shen and M. T. Kief, J. Appl. Phys., 79, 5008 (1996)

4. V.P. Nascimento, E.C. Passamani, A.D. Alvarenga, F. Pelegrini, A. Biondo, E. Baggio Saitovitch, J. Magn. Magn. Mater., 320, e272-e274 (2008)

5. D. Han, J. Zhu, J.H. Judy, J. Appl. Phys,. 81, 4996 (1997)
6. M. C. Cyrille, S. Kim, M. E. Gomez, J. Santamaria, Kannan M. Krishnan, and Ivan K. Schuller, Phys. Rev. B, 62, 3361-3367 (2000)

7. A. Maitre, D.Ledue, R. Patt, J. Magn. Magn. Mater., 324, 403-409 (2012)

8. Ch. Kittel, Phys. Rev., 73, 155 (1948)

9. J. C. Scott, J. Appl. Phys., 57, 3681 (1985)

10. A. A. Layadi, W. C. Cain, J. W. Lee, and J. O. Artman, IEEE Trans. Magn., MAG 23, 2993 (1987)

11. T. Ambrose and C. L. Chien, J. Appl. Phys., 79(8), 5920-5922 (1996)

12. T. Ambrose, C. L. Chien, J. Appl. Phys., 83, $6822-$ 6824 (1998)

13. H. Sang and Y. W. Du, C. L. Chien, J. Appl. Phys., 85, 4931-4933 (1999)

14. H. Xi, K. R. Mountfield, and R. M. White., J Appl. Phys., 87, 4367-4374 (2000)

15. T. Lin, C. Tsang, R. E. Fontana and J. K. Howard, IEEE Trans. Magn. MAG, 31(6), 2585 (1995)

16. H. N. Fuke, K. Saito, M. Yoshikawa, H. Iwasaki,and M. Sahashi, J. Appl. Phys., 75, 3679-3682 (1999)

17. J. Van Driel, F.R. de Boer, K.-M.H. Lenssen, R. Coehoorn, J. Appl. Phys., 88, 975-982 (2000)

18. A. P. Malozemoff, Phys. Rev. B, 37, 7673-7682 (1988)

19. K. O'Grady, L.E.Fernandez-Outon, G.VallejoFernandez J. Magn. Magn. Mater., 322, 883-899 (2010)

20. G Vallejo-Fernandez, L E Fernandez-Outon and K O'Grady, J. Phys. D: Appl. Phys., 41, 112001 (2008) 